Ann. Biol. anim. Bioch. Biophys., I968, 8 (3), 36r-37r.

\title{
ÉVOLUTION DES TENEURS EN ACIDES NUCLÉIQUES ET EN PROTÉINES DANS LES TISSUS DU RAT SOUMIS A UNE CARENCE ÉNERGÉTIQUE SÉVÈRE ET PROLONGÉE. CROISSANCE COMPENSATRICE
}

\author{
G. DURAND, G. FAUCONNEAU et Éliane PENOT \\ avec la collaboration technique de Noëlle BourgEAUX \\ Station centrale de Recherches de Nutrition, \\ Centre national de Recherches zootechniques, 78-Jouy-en-Josas \\ Station d'Étude des Métabolismes, \\ Centre de Recherches zootechniques et vétérinaires, 63 - Theix, près Clermont-Ferrand \\ Institut national de la Recherche agronomique
}

\section{SOMMAIRE}

L'apport énergétique de la ration allouée à des rats pubères, âgés de 9 semaines et pesant $275 \mathrm{~g}$, est réduit de telle façon que le poids des animaux diminue de $27 \mathrm{p}$. 100 en 28 jours. A ce stade une partie des animaux est sacrifiée et l'autre reçoit de nouveau, à volonté, une alimentation normale. Ces derniers animaux présentent une forte croissance compensatrice et recouvrent leur poids initial au bout de 5 jours, moment où ils sont sacrifiés à leur tour.

Les variations du nombre et de la taille des cellules du foie, des muscles des membres postérieurs, du corps éviscéré, sont mesurées respectivement par les variations de la teneur en ADN et du rapport Poids frais/ADN. Les variations des teneurs en ARN et en protéines sont considérées comme tests de l'activité cellulaire.

Les principaux résultats sont les suivants :

I. pendant la période de restriction

- le foie perd peu de cellules, le tiers de ses protéines et près de la moitié de son ARN,

- les muscles des membres postérieurs perdent très peu de cellules, très peu de protéines et près de la moitié de leur ARN,

- le corps éviscéré perd le cinquième de ses cellules, près de la moitié de son $\mathrm{ARN}$, mais peu de protéines ;

2. pendant la période de croissance compensatrice

- le foie et les muscles recouvrent leurs caractéristiques initiales.

- en revanche, pendant le court laps de temps que dure la croissance compensatrice, le corps éviscéré ne reconstitue que $29 \mathrm{p}$. Ioo des cellules, $79 \mathrm{p}$. Ioo de l'ARN et $73 \mathrm{p}$. Ioo des protéines disparues au cours de l'amaigrissement. 


\section{INTRODUCTION}

L'évolution des teneurs en acides nucléiques et en protéines des organes et tissus d'animaux perdant du poids a été surtout envisagée lorsque cette perte de poids était due au jeûne protéique (CAMPBEL, et KosTERLITZ, I948; MANDEL et coll., I949-I950-I954 ; SuMmERS et Fisher, I962). Les travaux de MANDEL en particulier ont permis de montrer que sous l'action d'un jeûne protéique de longue durée, entraînant chez des rats adultes une perte de poids vif de 40 à 45 p. Ioo, les quantités globales d'ADN contenues dans le foie, le rein et le muscle ne diminuaient pas, tandis qu'elles diminuaient notablement dans la rate, le pancréas et les surrénales. Dans tous les cas, les quantités d'ARN et de protéines subissaient une chute considérable.

Cependant, la carence en un élément nutritif indispensable, protéique, vitaminique ou autre, entraîne généralement une diminution de l'appétit de l'animal et, par conséquent, une diminution de l'ingestion d'éléments énergétiques (TERROINE, I964). Les effets du manque d'énergie risquent de venir s'ajouter à ceux de la carence originelle. C'est la raison pour laquelle une étude des effets propres à la restriction énergétique de la ration a été entreprise. Dans une première publication (DURAND et coll., I967), nous avons étudié les effets d'une restriction énergétique modérée sur les variations des teneurs des acides nucléiques et des protéines dans le foie, les muscles des membres postérieurs et le corps éviscéré du Rat en croissance.

Nous envisageons ici une étude des mêmes phénomènes au cours :

I. d'une période de restriction énergétique sévère et prolongée entraînant une perte de poids importante chez le Rat pubère;

2. de la période de croissance compensatrice qui succède à la phase de décroissance lorsque les animaux peuvent à nouveau s'alimenter à volonté en énergie.

Les variations des quantités d'ADN permettent de mesurer l'évolution du nombre de noyaux (BorviN et coll., I948) et, par extension de cellules, en appelant cellule l'ensemble d'un noyau et de la quantité moyenne de cytoplasme qu'il contrôle (DURAND et coll., I965). Le rapport poids frais/ADN varie comme la taille moyenne des cellules. Les rapports protéines/ADN et ARN/ADN sont considérés comme deux critères de 1'activité cellulaire.

\section{MATÉRIEL, ET MÉTHODES}

\section{Matériel animal}

Quatre lots de dix rats mâles Wiistar, dont les âges et les poids à l'abattage sont rapportés dans le tableau $\mathrm{I}$ et la figure $\mathrm{I}$, sont utilisés. Les animaux sont mis en expérience à l'âge de quatre semaines, pesant $7 \circ \mathrm{g} \pm 2 \mathrm{~g}$. Ils reçoivent ad libitum un régime semi-synthétique équilibré contenant $13 \mathrm{p}$. 100 de protéines $(\mathrm{N} \times 6,25)$. La composition du régime est rapportée dans le tableau 2. Les animaux du lot I sont abattus à $200 \mathrm{~g}$, ceux du lot II sont abattus à $275 \mathrm{~g}$.

Lorsque les animaux des lots III et IV ont atteint le poids de $275 \mathrm{~g}$, ils sont soumis à une restriction sévère de l'apport des éléments énergétiques de la ration (glucides et lipides); cette restriction est 
appliquée chaque jour de telle façon que le poids moyen des animaux décroisse linéairement et soit revenu au poids d'abattage des animaux du lot $\mathrm{I}$, soit $200 \mathrm{~g}$, en 28 jours. Ceci correspond à une perte de poids de $27 \mathrm{p}$. IOo. Lorsque ce résultat est acquis, les animaux du lot III sont abattus et ceux du

TABLEAU I

\begin{tabular}{l|r|r|r|r}
\hline \hline No des lots.......... & I & II & III & IV \\
\hline & & & & \\
\hline & & & & \\
Poids vif/rat (g) $\ldots \ldots \ldots \ldots$ & 200 & 275 & 200 & 278 \\
Age (jours) .......... & 50 & 63 & 91 & 96 \\
Nombre d'animaux ..... & 7 & 5 & 6 & 6 \\
\hline
\end{tabular}

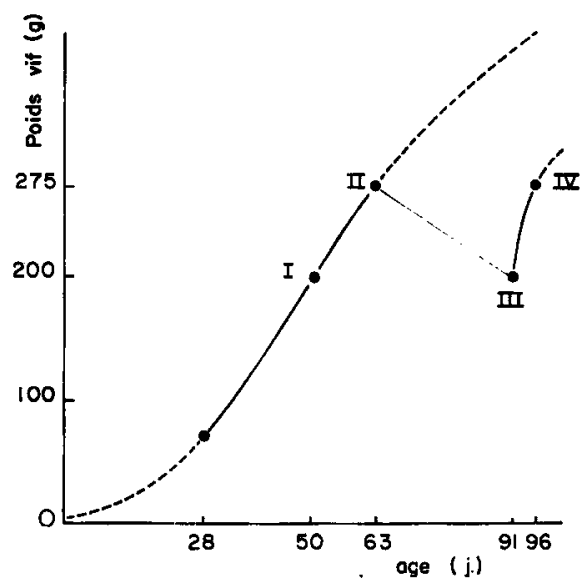

FIG. I. - Evolution de poids des animaux (en grammes) en fonction de l'age (en jours)

\section{TABLEAU 2}

Composition centésimale du régime distribué aux animaux en croissance

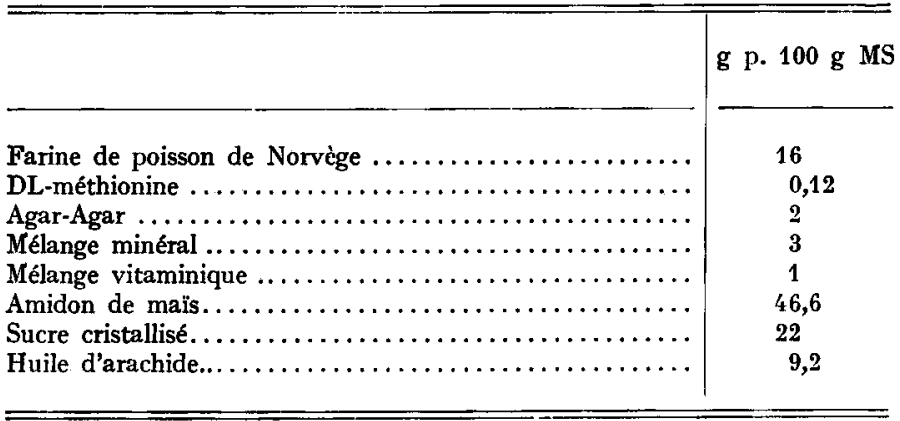

lot IV sont réalimentés à volonté ; ces derniers présentent une forte croissance compensatrice et sont abattus lorsque leur poids vif atteint de nouveau $275 \mathrm{~g}$, soit 5 jours plus tard.

A l'intérieur de chaque lot, seuls sont soumis aux analyses les animaux dont le poids s'écarte de moins de $5 \mathrm{p}$. roo du poids moyen. 
Croissance, décroissance et consommations alimentaires (fig. 2, tabl. 3).

Au cours de la croissance normale, la consommation est mesurée.

Pour amener le poids des animaux des lots III et IV de 275 à $200 \mathrm{~g}$, les animaux reçoivent chaque jour les quantités de protéines, minéraux et vitamines que consommeraient des animaux de même poids en croissance normale ; pour obtenir chaque jour la décroissance désirée $(2,7 \mathrm{~g}$ environ), les quantités de glucides et de lipides (sucre, amidon, huile d'arachide) doivent être réduites de 85 p. 100 par rapport aux quantités consommées par des animaux de même poids en croissance normale. Ceci revient à distribuer aux animaux restreints un régime à 39 p. Ioo de protéines $(\mathrm{N} \times 6,25)$.

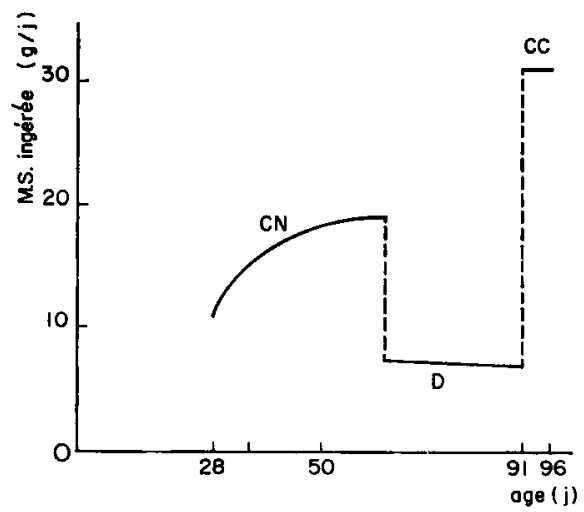

Frg. 2. - Consommation des animaux (en grammes de matière sèche par jour) en fonction de l'âge (en jours)

MS : Matière sèche $\quad \mathrm{CN}$ : Croissance normale $\quad \mathrm{D}$ : Décroissance $\mathrm{CC}$ : Croissance compensatrice.

TABLEAU 3

Consommation journalière et vitesse de croissance au cours de la croissance normale et de la croissance compensatrice

\begin{tabular}{|c|c|c|}
\hline & $\begin{array}{c}\text { Croissance } \\
\text { normale }\end{array}$ & $\begin{array}{c}\text { Croissance } \\
\text { compensatrice }\end{array}$ \\
\hline Poids vif $(g) \ldots \ldots \ldots \ldots \ldots$ & de 200 à 275 & de 200 à 278 \\
\hline $\begin{array}{l}\text { Consommation (g/jour) } \ldots \ldots \ldots \ldots \\
\text { Gain de poids/jour ............ } \\
\text { Indice de consommation } \ldots \ldots \ldots \ldots\end{array}$ & $\begin{array}{r}18,7 \\
5,8 \\
3,2\end{array}$ & $\begin{array}{r}31,7 \\
15,6 \\
2,0\end{array}$ \\
\hline
\end{tabular}

Au cours de la croissance compensatrice, les animaux du lot IV consomment $68 \mathrm{p}$. Ioo de plus que des animaux de même poids en croissance normale. La vitesse de croissance estimée en gain de poids vif par jour est 2,6 fois plus rapide.

Comportement et aspect des animaux.

Les animaux soumis à la réduction de l'apport énergétique reçoivent leur ration $(7 \mathrm{~g} \mathrm{MS} /$ jour environ) en une seule fois et l'ingèrent en quelques minutes. Leur état de santé ne semble pas affecté par ce régime.

Abattage et prélèvements.

Le mode d'abattage des animaux et le prélèvement des foies, muscles des membres postérieurs, et des corps éviscérés ont déjà été décrits (DuRAND et coll., 1967). 
Pour chaque lot, l'ensemble des foies, des muscles des membres postérieurs, et des corps éviscérés sans ces muscles sont séparément rassemblés. On obtient ainsi trois échantillons qui seront analysés.

\section{Méthodes analytiques}

L'ensemble des méthodes analytiques de dosage de l'azote et des acides nucléiques des diverses parties prélevées, ainsi que la composition globale des corps éviscérés ont déjà été décrites (DURAND et coll., I965).

\section{RÉSULTATS}

\section{Expression des résultats}

Les résultats exposés ci-dessous, concernant le corps éviscéré, ont été calculés en utilisant les données correspondant à la musculature des membres postérieurs et celles correspondant au corps éviscéré sans cette musculature.

Les quantités d'acides nucléiques sont exprimées par la somme en micromoles $(\mu \mathrm{M})$ de leurs bases constitutives.

Le résidu sec et dégraissé des diverses parties analysées contient essentiellement des protéines et sa teneur en azote est à peu près constante ( $148 \mathrm{mg} / \mathrm{g}$, soit 92,5 p. IOo de protéines $\mathrm{N} \times 6,25)$. La détermination du poids $\mathrm{SD}\left({ }^{1}\right)$ permet donc d'apprécier la teneur en protéines des fractions analysées.

FOIE (tabl. 4)

Action de la restriction. - Lorsque le poids vif des animaux diminue de 27 p. roo, le foie perd $4 \mathrm{I} \mathrm{p}$. Ioo de son poids frais, $32 \mathrm{p}$. Ioo de ses protéines et seulement Io $p$. roo de son capital d'ADN. Le rapport poids frais/ADN diminue de $34 \mathrm{p}$. roo et le rapport protéines/ADN de $25 \mathrm{p}$. 10o. La diminution de la teneur en ARN atteint 47 p. Ioo, entraînant une chute du rapport $A R N / A D N$ de $3, I$ à $I, 8$.

Action de la réalimentation. - Lorsque le poids vif des animaux du lot IV atteint de nouveau $275 \mathrm{~g}$, le poids frais et la teneur en protéines de leur foie sont l'un et l'autre supérieurs de ro p. Ioo à ce qu'ils étaient chez le lot II. Les quantités d'ADN et d'ARN sont pratiquement reconstituées et par conséquent, le rapport ARN/ADN retrouve sa valeur initiale, soit 3,I. Les rapports poids frais/ADN et protéines/ADN sont légèrement plus grands chez les animaux du lot IV que chez ceux du lot II.

\section{MUSCLES DES MEMBRES POSTÉRIEURS (tabl. 5)}

Action de la restriction. - Les muscles des membres postérieurs perdent I4 p. IOO de leur poids frais et 7 p. Ioo de leurs protéines. La quantité d'ADN diminue de 6 p. Ioo, la quantité d'ARN de 45 p. Ioo. Le rapport poids frais/ADN diminue de 9 p. roo, le rapport protéines/ADN n'est pas modifié et le rapport ARN/ADN passe de $I, 5$ à 0,9 .

(1) SD : Sec et dégraissé. 
Action de la réalimentation. - Lorsque le poids vif des animaux du lot IV atteint à nouveau $275 \mathrm{~g}$, le poids des muscles et leur teneur en protéines sont plus élevés que chez les animaux du lot II :

\section{TABLEAU 4}

Foie

Variations des teneurs en acides nucléiques et en protéines dans le foie du Rat pubère successivement : en croissance normale, en décroissance, en croissance compensatrice

\begin{tabular}{|c|c|c|c|c|}
\hline $\begin{array}{l}\text { No des lots } \ldots \ldots \ldots \ldots \ldots \ldots \ldots \ldots \ldots \ldots \\
\text { Poids vif }(\mathrm{g}) \ldots \ldots \ldots \ldots \ldots \ldots\end{array}$ & $\begin{array}{c}\mathrm{I} \\
200\end{array}$ & $\underset{275}{\text { II }}$ & $\begin{array}{l}\text { III } \\
200\end{array}$ & $\begin{array}{l}\text { IV } \\
278\end{array}$ \\
\hline Poids frais/rat $(\mathrm{g}) \ldots \ldots \ldots \ldots$ & 9,4 & 11,1 & 6,6 & 12,2 \\
\hline Poids frais $p .100$ poids vif...... & 4,7 & 4,1 & 3,3 & 4,4 \\
\hline Poids SD* $(\mathrm{g}) \ldots \ldots \ldots \ldots \ldots$ & 1,7 & 2,0 & 1,4 & 2,2 \\
\hline Poids SD p. 100 poids frais ..... & 18,0 & 18,2 & 21,0 & 17,9 \\
\hline $\operatorname{ADN}\left\{\begin{array}{l}\operatorname{lgSD}(\mu \mathrm{M} \text { bases) } \ldots \ldots \ldots \\
\text { total }(\mu \mathrm{M} \text { bases) }\end{array}\right.$ & $\begin{array}{l}37,1 \\
63,1\end{array}$ & $\begin{array}{l}37,1 \\
75,3\end{array}$ & $\begin{array}{l}49,2 \\
68,0\end{array}$ & $\begin{array}{l}33,8 \\
74,1\end{array}$ \\
\hline $\begin{array}{l}\text { Poids frais (mg)/ADN ( } \mu \mathrm{M} \text { bases).. } \\
\text { Protéines (mg)/ADN ( } \mu \mathrm{M} \text { bases) } . . \\
\text { ARN/ADN } \ldots \ldots \ldots \ldots \ldots \ldots \ldots \ldots \\
\text { ARN total }(\mu \mathrm{M} \text { bases) } \ldots \ldots \ldots \ldots .\end{array}$ & $\begin{array}{c}149 \\
24,9 \\
3,7 \\
233\end{array}$ & $\begin{array}{r}148 \\
24,9 \\
3,1 \\
231\end{array}$ & $\begin{array}{r}97 \\
18,8 \\
1,8 \\
122\end{array}$ & $\begin{array}{c}165 \\
27,4 \\
3,1 \\
228\end{array}$ \\
\hline
\end{tabular}

* SD : Sec et dégraissé.

\section{TABLEAU 5}

Muscles des membres postérieurs

Variations des teneurs en acides nucleiques et en protéines dans les muscles du Rat pubère successivement : en croissance normale, en décroissance, en croissance compensatrice

\begin{tabular}{|c|c|c|c|c|}
\hline $\begin{array}{l}\text { No des lots } \ldots \ldots \ldots \ldots \ldots \ldots \ldots \ldots \\
\text { Poids vif }(g) \ldots \ldots \ldots \ldots \ldots \ldots \ldots\end{array}$ & $\begin{array}{c}I \\
200\end{array}$ & $\begin{array}{c}\text { II } \\
275\end{array}$ & $\begin{array}{l}\text { III } \\
200\end{array}$ & $\begin{array}{l}\text { IV } \\
278\end{array}$ \\
\hline 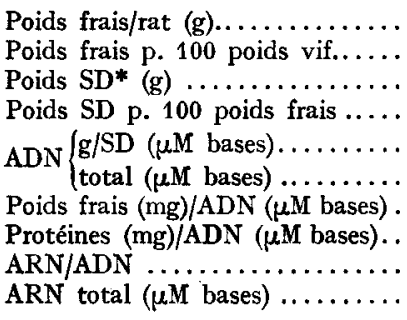 & $\begin{array}{r}17,2 \\
8,6 \\
3,3 \\
19,2 \\
11,3 \\
37,3 \\
461 \\
82 \\
2,0 \\
74,6\end{array}$ & $\begin{array}{r}21,1 \\
7,7 \\
4,3 \\
20,4 \\
10,1 \\
43,3 \\
488 \\
91,6 \\
1,5 \\
66,9\end{array}$ & $\begin{array}{r}18,1 \\
9,0 \\
4,0 \\
22,1 \\
10,2 \\
40,7 \\
445 \\
90,7 \\
0,9 \\
36,7\end{array}$ & $\begin{array}{r}22,8 \\
8,2 \\
4,5 \\
19,8 \\
9,3 \\
42,2 \\
540 \\
99,5 \\
1,7 \\
72,5\end{array}$ \\
\hline
\end{tabular}

* SD : Sec et dégraissé.

de 8 p. Ioo pour le poids frais et 6,5 p. Ioo pour la teneur en protéines. La quantité d'ADN, qui n'avait que très peu diminué au cours de la restriction, revient prati- 
quement à sa valeur initiale et la quantité d'ARN dépasse légèrement ce 'qu'elle était avant la restriction. Les rapports poids frais/ADN, protéines/ADN et ARN/ADN sont donc légèrement plus élevés après la réalimentation.

CORPS ÉVISCÉRÉ (tabl. 6).

I. Composition globale.

La composition globale (eau, lipides, protéines $\mathrm{N} \times 6,25$ et cendres) des quatre lots expérimentaux a été étudiée (tabl. $6 a$ ). Il apparaît que les corps éviscérés des

\section{TABLEAU 6 \\ Corps Éviscéré}

Évolution de la composition globale du corps éviscéré du Rat pubère successivement : en croissance normale, en décroissance, en croissance compensatrice Composition des gains et perte de poids au cours des differentes phases

a) Composition globale

\begin{tabular}{|c|c|c|c|c|}
\hline $\begin{array}{l}\text { No des lots } \ldots \ldots \ldots \ldots \ldots \ldots \ldots \\
\text { Poids vif }(g) \ldots \ldots \ldots \ldots \ldots \ldots \ldots \\
\text { Poids du corps éviscéré (g) } \ldots \ldots\end{array}$ & $\begin{array}{c}I \\
200 \\
162\end{array}$ & $\begin{array}{l}\text { II } \\
275 \\
224\end{array}$ & $\begin{array}{l}\text { III } \\
200 \\
164\end{array}$ & $\begin{array}{l}\text { IV } \\
278 \\
\mathbf{2 2 5}\end{array}$ \\
\hline $\begin{array}{l}\text { Eau }(\%) \ldots \ldots \ldots \ldots \ldots \ldots \ldots \ldots \\
\text { Lipides }(\%) \ldots \ldots \ldots \ldots \ldots \ldots \ldots \ldots \\
\text { Protéines } \mathrm{N} \times 6,25(\%) \ldots \ldots \ldots \ldots \\
\text { Cendres }(\%) \ldots \ldots \ldots \ldots \ldots \ldots \ldots\end{array}$ & $\begin{array}{r}65,3 \\
13,0 \\
18,0 \\
3,7\end{array}$ & $\begin{array}{r}59,1 \\
19,2 \\
18,1 \\
3,6\end{array}$ & $\begin{array}{r}64,8 \\
7,9 \\
22,7 \\
4,6\end{array}$ & $\begin{array}{r}58,9 \\
19,9 \\
17,6 \\
3,6\end{array}$ \\
\hline
\end{tabular}

b) Composition des gains ou de la perte de poids au cours des phases de croissance normale (CN), de décroissance (D) et de croissance compensatrice (CC)

\begin{tabular}{|c|c|c|c|c|c|c|}
\hline & \multicolumn{2}{|c|}{$\begin{array}{c}\mathrm{CN} \\
\text { de } 200 \text { à } 275 \mathrm{~g}\end{array}$} & \multicolumn{2}{|c|}{$\begin{array}{c}\text { D } \\
\text { de } 275 \text { à } 200 \mathrm{~g}\end{array}$} & \multicolumn{2}{|c|}{$\begin{array}{c}\mathrm{CC} \\
\text { de } 200 \text { à } 275 \mathrm{~g}\end{array}$} \\
\hline & Total (g) & P. 100 & Total (g) & P. 100 & Total (g) & P. 100 \\
\hline $\begin{array}{l}\text { Eau } \ldots \ldots \ldots \ldots \ldots \ldots \\
\text { Lipides } \ldots \ldots \ldots \ldots \ldots \\
\text { Protéines } \mathrm{N} \times 6,25 . \\
\text { Cendres............ }\end{array}$ & $\begin{array}{l}+26,6 \\
+22 \\
+\quad 11,4 \\
+\quad 2,1\end{array}$ & $\begin{array}{r}+42,9 \\
+\quad 35,3 \\
+\quad 18,4 \\
+\quad 3,4\end{array}$ & $\begin{array}{r}-26,1 \\
-\quad 30,1 \\
-\quad 3,4 \\
-\quad 0,5\end{array}$ & $\begin{array}{r}-43,0 \\
-\quad 50,2 \\
-\quad 5,6 \\
-\quad 0,8\end{array}$ & $\begin{array}{l}+\quad 26,3 \\
+\quad 31,8 \\
+\quad 2,5 \\
+\quad 0,6\end{array}$ & $\begin{array}{l}+\quad 43,1 \\
+\quad 52,1 \\
+\quad 4,1 \\
+\quad 1\end{array}$ \\
\hline
\end{tabular}

animaux du lot IV n'ont pas une composition très différente de ceux du lot II. Les résultats ont permis de calculer les compositions des gains et de la perte de poids au cours des trois phases expérimentales (croissance $\rightarrow$ décroissance $\rightarrow$ croissance compensatrice) (tabl; 6 b).

Au cours de la croissance normale, lorsque le poids vif des animaux augmente 
de 200 à $275 \mathrm{~g}$, le poids du corps éviscéré augmente de $62 \mathrm{~g}$. Ce gain de poids est constitué de 42,9 p. Ioo d'eau, de 35,3 p. Ioo de lipides, de I8,4 p. Ioo de protéines, et de 3,4 p. roo de cendres. Au cours de la décroissance, lorsque le poids vif est ramené de 275 à $200 \mathrm{~g}$, le poids du corps éviscéré est diminué de $60 \mathrm{~g}$. La perte de poids est constituée de 43,0 p. Ioo d'eau, de 50,2 p. Ioo de lipides, de 5,6 p. Ioo de protéines, et de $0,8 \mathrm{p}$. Ioo de cendres. Au cours de la croissance compensatrice qui ramène le poids vif des animaux légèrement au-dessus de $275 \mathrm{~g}$, le corps éviscéré reprend $6 \mathrm{I} \mathrm{g}$. Le gain de poids est constitué de 43, I p. Ioo d'eau, de 52,I p. I00 de lipides, de $4, I$ p. Ioo de protéines, et de I p. Ioo de cendres. Finalement, les corps éviscérés des lots II et IV ont une composition globale très voisine.

\section{2. Étude biochimique.}

a) Action de la restriction. - Au cours de la restriction, le corps éviscéré perd 27 p. Ioo de son poids frais et 8,4 p. Ioo seulement de ses protéines. Il perd en même temps 21 p. Ioo de son ADN et 47 p. Ioo de son ARN. Le rapport poids frais/ADN est diminué de $7 \mathrm{p}$. roo, le rapport protéines/ADN est augmenté de $22 \mathrm{p}$. Ioo, et le rapport $A R N / A D N$ est diminué de 32 p. Ioo.

\section{TABLEAU 7}

\section{Corps éviscéré}

Variations des teneurs en acides nucléiques et en protéines dans le corps éviscéré du Rat pubère successivement : en croissance normale, en décroissance, en croissance compensatrice

\begin{tabular}{l|c|c|c}
\hline \hline & & & \\
No des lots $\ldots \ldots \ldots \ldots \ldots \ldots \ldots$ \\
Poids vif $(\mathrm{g}) \ldots \ldots \ldots \ldots \ldots \ldots \ldots$ \\
\end{tabular}

* SD : Sec et dégraissé.

b) Action de la réalimentation. - Lorsque les animaux du lot IV atteignent à nouveau le poids des animaux du lot II, le corps éviscéré a reconstitué la totalité du poids frais, $73 \mathrm{p}$. I00 des protéines, $79 \mathrm{p}$. Ioo de l'ARN, 29 p. I00 seulement de l'ADN perdus au cours de l'amaigrissement. Le rapport poids frais/ADN est plus grand de 20 p. Ioo dans le corps éviscéré des animaux du lot IV, comparés à ceux du lot II ; de même, le rapport protéines/ADN est supérieur à ce qu'il était initialement, contrairement au rapport ARN/ADN redevenu normal. 


\section{DISCUSSION}

\section{A. - Action de la restriction énergétique}

Lorsque des rats mâles âgés de 9 semaines sont soumis à une réduction sévère des éléments énergétiques de la ration telle que les animaux perdent $27 \mathrm{p}$. Ioo de leur poids vif en 28 jours, le foie, les muscles et le corps éviscéré réagissent de manières diverses:

- le poids frais du foie diminue plus que l'ensemble du corps de l'animal. Les teneurs en protéines et en ARN de l'organe diminuent fortement. En revanche, on peut considérer que le nombre de ses cellules demeure à peu près constant, la différence observée, soit Io $\mathrm{p}$. Ioo, ne paraissant pas significative.

Les muscles se révèlent très résistants à la carence énergétique : ainsi, lorsque le poids vif des animaux est diminué de $27 \mathrm{p}$. roo, les muscles des membres postérieurs ne perdent que I4 p. Ioo de leur poids frais. De plus, les pertes en protéines (7 p. roo) ne peuvent guère être prises en considération, pas plus que la diminution du nombre de cellules ( 6 p. I0o). Dans une étude concernant des muscles de rats adultes soumis à un jeûne protéique prolongé tel que le poids vif des animaux diminuait de 4I p. IOO, MANDEL, et coll. (2I949) constataient une perte d'ADN voisine de 9 p. Ioo; cette faible diminution n'était pas non plus considérée comme significative par les auteurs.

Il ressort donc des travaux de MANDEL et des nôtres que le nombre des noyaux musculaires manifeste une remarquable stabilité face à une malnutrition protéique et/ou énergétique très sévère. Il semble dès lors, que des phases de décroissance périodiques (femelles en lactation, disettes saisonnières) ne puissent pas faire varier notablement le nombre de noyaux des fibres musculaires. Seule la teneur en ARN est durement touchée par la carence.

Pendant la période de restriction, le corps éviscéré perd à peu près la même quantité d'eau, plus de lipides et moins de protéines qu'il en avait gagné au cours de la période de croissance normale où le poids des animaux était passé de 200 à $275 \mathrm{~g}$. En même temps, on constate une importante perte d'ADN (2I p. Ioo), donc de cellules. D'après ce que nous avons vu plus haut, une faible part seulement de ce phénomène peut être imputée au tissu musculaire. C'est probablement dans les tissus adipeux (épididymaire, périrénal, mésentérique, etc.), visiblement très amoindris, que la perte de cellules est la plus marquée. Cependant, il est également probable que d'autres tissus soient concernés. Un travail est actuellement en cours, qui doit permettre d'éclaircir ce problème. D'autre part, aptès l'amaigrissement, le rapport poids frais/ADN est peu modifié. On en déduit qu'il disparaît proportionnellement autant de grandes que de petites cellules. Par ailleurs, les unes (cellules adipeuses) et les autres (cellules de petite taille) contiennent peu de protéines, ce qui explique la modicité $(8,4 \mathrm{p}$. Ioo) de la diminution protéique globale du corps éviscéré. La perte d'ARN total au cours de 1'amaigrissement est considérable (47 p. 100); elle est due en partie à la perte de l'ARN des cellules mortes et à sa diminution dans les cellules encore vivantes. La diminution du rapport ARN/ADN montre que la perte d'ARN à l'intérieur des cellules vivantes autres que les cellules musculaires est certainement inférieure à $30 \mathrm{p}$. Ioo.

Annales de Biologie animale. - 1968. 


\section{B. - Action de la réalimentation}

Les animaux amaigris puis à nouveau alimentés à volonté présentent une croissance compensatrice impressionnante avec un gain de poids quotidien de $\mathrm{I}_{5}, 6 \mathrm{~g}$. Ils recouvrent donc leur poids initial en un temps extrêmement court. Ce dernier suffit pour que le foie reconstitue son capital initial d'acides nucléiques et de protéines. I1 en est de même pour les muscles, pour eux d'autant plus aisément qu'ils n'avaient guère été touchés par la carence énergétique.

Il en va autrement du corps éviscéré qui ne retrouve que $29 \mathrm{p}$. Ioo de l'ADN, $73 \mathrm{p}$. Ioo des protéines et $79 \mathrm{p}$. Ioo de 1'ARN précédemment perdus. La reprise de la synthèse de $1^{\prime} A R N$ et des.protéines est donc plus rapide que la reprise de la synthèse de 1'ADN. La période de réalimentation qui a permis aux animaux de reprendre leur poids vif initial est de courte durée ( 5 jours). Nous ne pouvons pas dire si sur une durée plus longue, le capital cellulaire ne pourrait pas être reconstitué complètement. Peutêtre peut-on penser que les cellules de certains tissus qui ont survécu à la restriction commencent par synthétiser activement de 1'ARN et des protéines avant de se diviser à nouveau pour reconstituer les tissus détruits et que la brève durée de la réalimentation nous a permis de saisir seulement le début de la phase active de régénération des tissus morts. Un tel processus serait conforme à ce que l'on sait de la régénération du foie après hépatectomie partielle, au cours de laquelle l'augmentation de la synthèse de 1'ARN (MCARDLE et CREASER, I963; CATER et coll., I956), et des protéines (GuINos, I958 a) précède la synthèse de 1'ADN.

Comparaison de corps éviscérés d'animaux parvenus au poids de $200 \mathrm{~g}$ par des voies différentes.

Lorsque l'on compare des animaux qui ont atteint le poids de $200 \mathrm{~g}$, soit à la suite d'une croissance normale, soit à la suite d'une croissance ralentie par réduction modérée de l'apport énergétique de la ration (DuRAND et coll., r967), soit à la suite de la décroissance décrite ci-dessus, on constate que les quantités d'ADN contenues dans les corps éviscérés des trois types d'animaux sont peu différentes (respectivement $604,574,548 \mu \mathrm{M}$-bases). Comment, suivant les cas, l'ADN est-il réparti entre les tissus?

Si l'on admet 1'hypothèse que la concentration en ADN de l'ensemble de la musculature est la même que celle mesurée dans les muscles des membres postérieurs, il apparaît que la quantité totale d'ADN dans la musculature est un peu plus élevée $(+9$ p. IOo) chez les animaux amaigris, plus faible (-20 p. IOo) chez les animaux à croissance ralentie, que chez les animaux à croissance normale.

Cependant, nous ne savons pas encore comment les régimes alimentaires imposés ont induit la distribution du capital d'ADN non musculaire entre les différents autres tissus (peau, tissus adipeux, squelette). 


\section{SUMMARY}

\section{CHANGES IN NUCLEIC ACID AND PROTEIN CONTENTS IN TISSUES OF RATS SUBMITTED TO SEVERE AND PROLONGED DEPRIVATION OF ENERGY. COMPENSATORY GROWTH.}

The supply of energy of the ration of pubescent rats 9 weeks old and weighing $275 \mathrm{~g}$ was reduced in such a way that their weight diminished by 27 per cent in 28 days. At this stage some of them were killed and the others were again fed to appetite on a normal feed. These latter animals showed great compensatory growth and recovered to their initial weight at the end of 5 days, when they in their turn were killed.

Differences in number and size of cells of liver, muscles of the hind limb and eviscerated body were estimated from content of DNA and ratio of fresh weight to DNA, respectively. Differences in contents of RNA and protein were considered as indications of activity of cells.

The principal results showed that :

I. during the period of restriction

- the liver lost few cells, a third of its proteins and about a half of its RNA,

- the muscles of the hind limbs lost very few cells, very little protein and about half trait RNA,

- the eviscerated body lost a fifth of its cells and about half of its RNA but little of its proteins;

2. during the period of compensatory growth

- the liver and muscles regained their original characteristics,

- in contrast, during the short lapse of time which compensatory growth lasted, the eviscerated body regained only 29 per cent of the cells, 79 per cent of the RNA and 73 per cent of the proteins which disappeared during loss of weight.

\section{RÉFÉRENCES BIBLIOGRAPHIQUES}

BoIvin A., VRENDLY R., VENDRELY C., I948. L'acide désoxyribonucléique du noyau cellulaire, dépositaire des caractères héréditaires; arguments d'ordre analytique. C. R. Acad. Sci., 226, 106i-1062.

Campbelt R. M., Kosteritiz H. W., I948. The effects of dietary protein on the turnover of phospholipids, ribonucleic acid, and desoxyribonucleic acid in the liver. J. Biol. Chem., 175, 989-990.

Cater D. B., Holmes B. E., MeE L. K., 1956. Cell division and nucleic acid synthesis in the regenerating liver of the rat. Acta radiol., 46, 655-667.

Durand G., Fauconneau G., Penot Éliane, 1965. Étude biochimique de la croissance de l'intestin grêle du foie et de la carcasse du Rat ; rôles respectifs de la multiplication et du grandissement cellulaires. Ann. Biol. anim. Bioch. Biophys., 5, 163-187.

Durand G., Fauconneau G., Penot Éliane, i 967 . Croissance des tissus du Rat et réduction de l'apport énergétique de la ration ; influence sur la teneur en acides nucléiques. Ann. Biol. anim. Bioch. Biophys., 7 , (sous presse).

GLINoS A. D., I958 a. Liver regeneration and liver function. In "Liver function ", A Symposium on Approches to the quantitative description of liver function (BRAUER R. W., ad.). Am. Inst. Biol.Sci. Publ.n $\mathrm{n}^{\circ}$, Washington $D$. C., 425-43I.

Mandel P., JacoB M., Mandel L., I 949. Action du jeûne protéique prolongé sur les deux acides nucléiques du muscle chez le Rat. C. R. Soc. Biol., 143, 536-539.

Mandel P., Jacob M., Mandel L., 1950. Étude sur le métabolisme des acides nucléiques. I. Action du jeûne prolongé sur les deux acides nucléiques du foie, du rein et du cerveau. Bull. Soc. Chim. Biol., 32,80-88.

Mandel P., Jaсов M., Mandel L., I954. Étude du jeûne protéique prolongé sur les acides pentos nucléique et désoxypentosenucléique du pancréas et des surrénales. C. R. Acad. Sci., 238, 288.

MCARDLE A. H., CREASER E. H., I963. Nucleoproteins in regenerating rat liver. I. Incorporation of ${ }^{32} \mathrm{P}$ into the ribonucleic acid of liver during early stages of regeneration. Biochem. Biophys. Acta, 68, $56 \mathrm{I}-568$.

Summers J. D., Fisher H., I96z. Nutritional requirements of the protein-depleted chicken. II. Effect of different protein depletion regimens on nucleic acids and enzyme activity in the liver. $J . N u l r ., 76,187^{-}$ 198.

Terroine T., rg64. Le contrôle vitaminique des activités enzymatiques. Ann. Nut. Alim., 18, C87-C86. 